EEESTI NSV TEADUSTE AKADEEMIA TOIMETISÊD. FOOSIKA * MATEMAATIKA

ИЗВЕСТИЯ АКАДЕМИИ НАУК ЭСТОНСКОИ ССР. ФИЗИКА * МАТЕМАТИКА PROCEEDINGS OF THE ACADEMY OF SCIENCES OF THE ESTONIAN SSR. PHYSICS * MATHEMATICS

$1986,35,1$

\title{
УСЛОВИЯ ДИСКРЕТНОЙ УСТОИЧИВОСТИ ОБЩЕЙ ЗАДАЧИ СТОХАСТИЧЕСКОГО ПРОГРАММИРОВАНИЯ С РЕШАЮЩИМИ ФУНКЦИЯМИ
}

\author{
(Представил Н. Алумяэ)
}

\section{1. Введение}

Существуют классы экстремальных задач, в которых решение ищется в виде измеримой вектор-функции от случайного параметра. К таковым относятся, в частности, задачи стохастического программирования с решающими функциями $[1,2]$ и задачи двухэтапного стохастического программирования в статической постановке $\left[{ }^{3}\right]$. Одна возможность приближенного решения подобных задач заключается в их дискретизации, т. е. в замене данной задачи последовательностью задач в конечномерных пространствах - заменой интегралов суммами и бесконечного числа ограничений конечным. Для обоснования такой замены надо выяснить условия, гарантирующие сходимость соответствующей последовательности решений к решению исходной задачи, т. е. условия дискретной устойчивости.

При приближенном решении интегральных и дифференциальных уравнений дискретизационный метод подробно изучен (см., напр., $\left.\left[{ }^{4}\right]\right)$. Применительно к приближенному решению экстремальных задач такой подход, по-видимому, был впервые предложен Дж. У. Даниэлем (см., напр., $\left.\left[{ }^{5}\right]\right)$ и развит дальше в $\left[{ }^{6-8}\right]$.

В настоящей статье для приближенного решения экстремальной задачи с интегральной целевой функцией и с интегральными и вероятностными ограничениями исходят из общей схемы дискретной аппроксимации экстремальных задач в функциональных пространствах. При этом учитывается, что задача, рассматриваемая в данной статье, имеет некоторые особенности. Так, например, при дискретизации экстремальной задачи в пространстве $L^{p}$ мы вынуждены использовать интегральный проектор, который в общем не проектирует множество ограничений исходной задачи в множество ограничений дискретизированной задачи. По этой и другим причинам прямое перенесение условий из $\left[{ }^{5-8}\right]$ в общем затруднительно и мы должны их модифицировать.

Проблеме устойчивости в том или ином смысле задачи стохастического программирования посвящено немало работ (см., напр., $\left[{ }^{9-12}\right]$. В этих трудах, за исключением $\left[{ }^{12}\right]$, использован теоретико-вероятностный подход. Например, в $\left[{ }^{10}\right]$ разбиение основного пространства элементарных событий осуществляется с помощью конечной $\sigma$-подалгебры, на элементах которой искомая функция считается кусочно постоянной. В настоящей работе используется функционально-аналитический подход. Предполагая только существование дискретных мер, в некотором смысле сходящихся к вероятностной мере исходной задачи, задаются 
условия; гарантирующие устойчивость дискретной аппроксимации. От плотности вероятностной меры требуется интегрируемость по Риману, ибо без этого путем варьирования значений плотности в конечном числе гочек можно получить расходящийся процесс аппроксимации. По этой же причине, по-видимому, и в $\left[{ }^{12}\right]$ нужно понимать интегрируемость в смысле Римана.

\section{2. Постановка задачи}

Пусть имеется случайный вектор $\xi=\xi(\omega)$ со значениями в измеримом пространстве $\left(R^{s}, B\right)$ с индуцированной вероятностной мерой $\mu$ с носителем $G \subset R^{s}$. Пусть на $R^{r} \times R^{s}$ заданы числовые функции $F_{k}(x, \xi)$, $k=0,1,2, \ldots, m, m+1,1=\overline{0, m+1}$. Рассмотрим задачу:

минимизировать функционал

$$
f_{0}(x)=\int_{G} F_{0}(x(\xi), \xi) \mu(d \xi)
$$

на множестве $X=Q \cap S$, задаваемом ограничениями

$$
\begin{gathered}
Q=\left\{x(\xi) \mid f_{k}(x)=\int_{G} F_{k}(x(\xi), \xi) \mu(d \xi) \leqslant 0, k=\overline{1, m}\right\}, \\
S=\left\{x(\xi) \mid F_{m+1}(x(\xi), \xi) \leqslant 0 \text { при почти всех (п. в.) } \xi \in G\right\} .
\end{gathered}
$$

Решение $x(\xi)$ задачи (1)-(3) будем искать в рефлексивном пространстве $L^{p}(\mu), 1<p<\infty$, суммируемых со степенью $p$ функций.

Мы не будем здесь выяснять условий существования решения в задаче $(1)-(3)$. Отметим только, что налагаемые на функции $F_{k}(x, \xi)$, $k=\overline{0, m+1}$, и меру $\mu$ условия, гарантирующие дискретную устойчивость, обеспечивают и существование решения в задаче (1)-(3).

Пусть дискретные меры $\mu_{n} ; \mu_{n}=\left\{\left(\xi_{i n}, \mu_{i n}\right), \xi_{\text {in }} \in G, i=\overline{1, n}\right\}$, $\mu_{i n}>0, i=\overline{1, n}, \sum_{i=1}^{n} \mu_{i n}=1$, слабо сходятся к вероятностной мере $\mu$, т. е.

$$
\lim _{n \rightarrow \infty} \sum_{i=1}^{n} h\left(\xi_{i n}\right) \mu_{i n}=\int_{G} h(\xi) \mu(d \xi)
$$

для любой непрерывной ограниченной на $G$ функции $h(\xi)$.

Рассмотрим вместо (1)-(3) следующую задачу: минимизировать функцию

$$
f_{0 n}\left(x_{n}\right)=\sum_{i=1}^{n} F_{0}\left(x_{i n}, \xi_{i n}\right) \mu_{i n}
$$

на множестве $X_{n}=Q_{n} \cap S_{n}$, задаваемом условиями

$$
\begin{gathered}
Q_{n}=\left\{x_{n} \mid f_{k n}\left(x_{n}\right)=\sum_{i=1}^{n} F_{k}\left(x_{i n}, \xi_{i n}\right) \mu_{i n} \leqslant 0, k=\overline{1, m}\right\}, \\
S_{n}=\left\{x_{n} \mid F_{m+1}\left(x_{i n}, \xi_{i n}\right) \leqslant 0, i=\overline{1 ; n}\right\} .
\end{gathered}
$$

Здесь и далее $x_{n}=\left\{x_{1 n}, \ldots, x_{n n}\right\}$, а $\xi_{i n}, \overline{i=1}, n$, суть точки меры $\mu_{n}$.

Решение задачи $(1 n)-(3 n)$ будем искать в конечномерном пространстве $l_{n} p\left(\mu_{n}\right)$ с нормой

$$
\left\|x_{n}\right\|_{n}=\left(\sum_{i=1}^{n}\left|x_{i n}\right|^{p} \mu_{i n}\right)^{1 / p}
$$


Определим между пространствами $L^{p}(\mu)$ и $l_{n}^{p}\left(\mu_{n}\right) \quad(n \in N=$ $=\{1,2,3, \ldots\})$ систему связывающих операторов $\mathfrak{T}=\left(p_{n}\right)$, полагая $p_{n}: L^{p}(\mu) \rightarrow l_{n}^{p}\left(\mu_{n}\right)$,

$$
p_{n} x(\xi)=\left(\mu\left(A_{\text {in }}\right)^{-1} \int_{A_{t n}} x(\xi) \mu(d \xi), i=\overline{1, n}\right)
$$

для некоторой совокупности множеств $A_{i n}, i=1,2, \ldots, n, \mu\left(A_{i n}\right)>0$ таких, что $\xi_{i n} \in A_{i n}, \bigcup_{i=1}^{n} A_{i n}=S, A_{i n} \cap A_{j n}=\varnothing \quad$ при $i \neq j, \operatorname{diam} A_{i n} \rightarrow 0$ при $n \rightarrow \infty$ и $\left|\mu_{\text {in }} \mu\left(A_{\text {in }}\right)^{-1}-1\right| \rightarrow 0$ при $n \rightarrow \infty$.

Аналогично определим систему связывающих операторов $\mathbb{Q}=\left(q_{n}\right)$ для пространств $L^{q}(\mu), l_{n}^{q}\left(\mu_{n}\right)(n \in N)$, где $1 / p+1 / q=1$.

Пусть $C(G)$ - пространство непрерывных на $G$ функций. Для пространств $C(G)$ и $l_{n}^{p}\left(\mu_{n}\right)$ определим помимо (6) систему связывающих операторов вида

$$
p^{\prime} x(\xi)=\left(x\left(\xi_{i n}\right), i=\overline{1, n}\right),
$$

где $\xi_{1 n}, \ldots, \xi_{n n}-$ точки меры $\mu_{n}$.

Системы связывающих операторов $\mathfrak{T}$ и $\mathfrak{f}^{\prime}$ эквивалентны в том смысле, что для любого $x \in L^{p}$

$$
\left\|p_{n} x-p_{n}^{\prime} x\right\|_{n} \rightarrow 0 \text { при } n \rightarrow \infty
$$

(см., напр., $\left.\left[{ }^{4}\right]\right)\left(\mathcal{S}^{\prime}\right.$ расширяется на $L^{p}$ с сохранением линейности и свойства $\left.\left\|p_{n} x\right\|_{n \rightarrow\|x\|} \forall x \in L^{p}\right)$.

Под парой $\left\{f,\left(f_{n}\right)\right\}$ будем понимать функционал $f$ с областью определения $D(f) \subseteq L_{p}$ и последовательность функций $\left(f_{n}\right)$ с областями определения $D\left(f_{n}\right) \subseteq l_{n}^{p}$.

Последовательность $\left(x_{n}\right), x_{n} \in l_{n}{ }^{p} \quad(n \in N)$, дискретно сходится к $x \in L^{p}$, если при $n \rightarrow \infty$

$$
\left\|x_{n}-p_{n} x\right\|_{n} \rightarrow 0 \text {. }
$$

Эту сходимость обозначим через $x_{n} \rightarrow x$.

Последовательность $\left(x_{n}\right), x_{n} \in l_{n}^{p}(n \in N)$, дискретно слабо сходится к $x \in L^{p}$, если $x_{n}(n \in N)$ и $x$, рассматриваемые как элементы пространств $\left[l_{n}^{p}\right]^{* *}(n \in N)$ и $\left[L^{p}\right]^{* *}$ соответственно, удовлетворяют соотношению

$$
\left\langle y_{n}, x_{n}\right\rangle \rightarrow\langle y, x\rangle
$$

для любой дискретно сходящейся последовательности функционалов: $y_{n} \rightarrow y, y_{n} \in l_{n}^{q}, y \in L^{q}$. Здесь $\langle y, x\rangle-$ значение линейного функционала $y$ в точке $x$.

Дискретную слабую сходимость будем обозначать через $x_{n} \rightarrow x$.

Будем говорить, что пара $\left\{f,\left(f_{n}\right)\right\}$ дискретно полунепрерывна снизу (сверху), если при $x_{n} \rightarrow x$ имеет место

$$
f(x) \leqslant \frac{\lim }{n \rightarrow \infty} f_{n}\left(x_{n}\right)
$$

(соответственно

$$
\left.f(x) \geqslant \varlimsup_{n \rightarrow \infty} f_{n}\left(x_{n}\right)\right) .
$$

Если дискретную сходимость элементов заменить их дискретной слабой сходимостью, то при выполнении вышеуказанных условий пара $\left\{f,\left(f_{n}\right)\right\}$ называется дискретно слабо полунепрерывной снизу (сверху). 


\section{3. Условия устойчивости дискретной аппроксимации}

Обозначим оптимальное значение задачи (1)-(3) через $f^{*}$ (соответственно задачи $(1 n)-(3 n)$ через $\left.f_{n}{ }^{*}\right)$.

Пусть задачи $(1)-(3)$ и $(1 n)-(3 n)(n \in N)$ разрешимы. Обозначим их решения через $x^{*} \equiv x^{*}(\xi)$ и $x_{n}{ }^{*}(n \in N)$ соответственно.

Наша основная цель - выяснить достаточные условия, налагаемые на функции $F_{k}(x, \xi), k=\overline{0, m+1}$, и на меру $\mu$, гарантирующие устойчивую аппроксимацию задачи (1)-(3) последовательностью задач $(1 n)-(3 n)$ как по оптимальному значению, так и по решению. При этом основными предположениями являются слабая сходимость последовательности дискретных мер $\mu_{n}$ к вероятностной мере $\mu$, которая должна иметь интегрируемую по Риману плотность и ограниченный носитель. Введем следующие условия:

У1) носитель $G$ вероятностной меры $\mu$ - ограниченное множество,

У2) вероятностная мера $\mu$ имеет интегрируемую по Риману плотность,

У3) функция $F(x, \xi)$ непрерывна по $(x, \xi)$, выпукла и дифференцируема по $x$ при всех $\xi$, и $F^{\prime}{ }_{x}(x, \xi)$ непрерывна по $(x, \xi)$,

У4) при некотором $k \in\{0,1, \ldots, m\}$ выполнено неравенство

$$
F_{k}(x, \xi) \geqslant a_{k}(\xi)+\alpha_{k}|x|^{p},
$$

где $\alpha_{k}<0$ и $a_{k}(\xi)$ является интегрируемой по Риману функцией, $a_{k}: G \rightarrow(-\infty, 0]$.

Обозначим $f(x)=\int_{G} F(x(\xi), \xi) \mu(d \xi), f_{n}\left(x_{n}\right)=\sum_{i=1}^{n} F\left(x_{i n}, \xi_{i n}\right) \mu_{i n}$.

Л е м м а 1. Если дискретные меры $\mu_{n}$ слабо сходятся $\kappa$ вероятностной мере $\mu$ и выполнены условия У1-У3, то пара $\left\{f(x),\left(f_{n}\left(x_{n}\right)\right)\right\}$ дискретно полунепрерывна сверху и дискретно слабо полунепрерывна снизу. Д ок а з а те льс т в о. Из условия УЗ получаем

$$
\begin{aligned}
& \sum_{i=1}^{n} F\left(x_{i n}, \xi_{i n}\right) \mu_{i n}-\int_{G} F(x(\xi), \xi) \mu(d \xi) \leqslant \\
\leqslant & \sum_{i=1}^{n}\left(F^{\prime}{ }_{x}\left(x_{i n}, \xi_{i n}\right), x_{i n}-p_{n}^{\prime} x(\xi)\right) \mu_{i n}+ \\
+ & \sum_{i=1}^{n} F\left(p^{\prime}{ }_{n} x(\xi), \xi_{i n}\right) \mu_{i n}-\int_{G} F(x(\xi), \xi) \mu(d \xi) .
\end{aligned}
$$

Ввиду условий У1 и У2 мы вправе использовать связывающие операторы $p^{\prime}{ }_{n}$ вида (6') для функции $x(\xi)$, так как по условию У2 для любого $A$ из $B$ имеем $\mu\left(A^{0}\right)=\mu(A)=\mu(\bar{A})$, где $A^{0}$ и $\bar{A}-$ внутренность и замыкание множества $A$ соответственно. Из этого факта вытекает, что мы вправе искать и функцию $x(\xi)$ как интегрируемую по Риману функцию (подробнее о таком сужении меры см. в $\left[{ }^{13}\right]$ ). Тогда

$$
\begin{gathered}
\left|\sum_{i=1}^{n}\left(F_{x}^{\prime}\left(x_{i n}, \xi_{i n}\right), x_{i n}-p^{\prime}{ }_{n} x(\xi)\right) \mu_{i n}\right| \leqslant \\
\leqslant\left(\sum_{i=1}^{n}\left|F^{\prime}{ }_{x}\left(x_{i n}, \xi_{i n}\right)\right|^{q} \mu_{i n}\right)^{1 / q}\left(\sum_{i=1}^{n}\left|x_{i n}-p_{n}^{\prime} x(\xi)\right|{ }^{p} \mu_{i n}\right)^{1 / p} .
\end{gathered}
$$

В силу ограниченности $F^{\prime}{ }_{x}(x, \xi)$ и дискретной сходимости $x_{n} \rightarrow x$ для любого $\varepsilon>0$ найдется натуральное $n_{1}$ такое, что при $n \geqslant n_{1}$ последнее выражение не превосходит $\varepsilon / 2$. Слабая сходимость дискретных мер $\mu_{n}$ к вероятностной мере $\mu$ вместе с условиями $У 1-У 3$ гараңтирует при всех $n$, начиная с некоторого $n_{2}$, выполнение неравенства 


$$
\left|\sum_{i=1}^{n} F\left(p_{n}^{\prime} x(\xi), \xi_{i n}\right) \mu_{i n}-\int_{G} F(x(\xi), \xi) \mu(d \xi)\right|<\varepsilon / 2 .
$$

Взяв $n \geqslant \max \left(n_{1}, n_{2}\right)$, получим дискретную полунепрерывность сверху пары $\left\{f(x),\left(f_{n}\left(x_{n}\right)\right)\right\}$.

Докажем дискретную слабую полунепрерывность снизу. Пусть $x_{n} \longrightarrow x$. Рассмотрим разность

$$
\begin{aligned}
& \int_{G} F(x(\xi), \xi) \mu(d \xi)-\sum_{i=1}^{n} F\left(x_{i n}, \xi_{i n}\right) \mu_{i n}= \\
= & \int_{G} F(x(\xi), \xi) \mu(d \xi)-\sum_{i=1}^{n} F\left(x\left(\xi_{i n}\right), \xi_{i n}\right) \mu_{i n}+ \\
+ & \sum_{i=1}^{n} F\left(x\left(\xi_{i n}\right), \xi_{i n}\right) \mu_{i n}-\sum_{i=1}^{n} F\left(x_{i n}, \xi_{i n}\right) \mu_{i n} \leqslant \\
\leqslant & R_{n}+\sum_{i=1}^{n}\left(F_{x}^{\prime}\left(x\left(\xi_{i n}\right), \xi_{i n}\right), x\left(\xi_{i n}\right)-x_{i n}\right) \mu_{i n},
\end{aligned}
$$

где через $R_{n}$ для краткости обозначена разность

$$
R_{n}=\int_{G} F(x(\xi), \xi) \mu(d \xi)-\sum_{i=1}^{n} F\left(x\left(\xi_{i n}\right), \xi_{i n}\right) \mu_{i n} .
$$

Дискретная слабая сходимость $x_{n} \rightarrow x$ в настоящем случае эквивалентна выполнению следующих двух условий:

1) $\left\|x_{n}\right\|_{n} \leqslant$ const,

2) $\left\langle q_{n}^{\prime} y, x_{n}\right\rangle \rightarrow\langle y, x\rangle$ при $n \rightarrow \infty$,

для любого $y$ из $L^{q}(\mu)$. (Предположение о существовании интегрируемой по Риману плотности дает нам право использовать связывающий оператор $p_{n}^{\prime}$ вида $\left.\left(5^{\prime}\right)\right)$. Ввиду непрерывности функции $F_{x}^{\prime}(x, y)$ по $(x, y)$ можно для $y(\xi)=F_{x}^{\prime}(x(\xi), \xi)$ использовать систему связывающих операторов $q_{n}^{\prime}$ вида $\left(5^{\prime}\right)$. Следовательно, операторы $q_{n}^{\prime} F^{\prime}{ }_{x}\left(p^{\prime}{ }_{n} x\right)=$ $=F_{n x}^{\prime}\left(p_{n}^{\prime} x\right)=\left(F_{x}^{\prime}\left(x\left(\xi_{i n}\right), \xi_{i n}\right), i=\overline{1, n)} \quad(n \in N)\right.$ удовлетворяют условию 2) леммы. Условие 1) выполнено в силу дискретной слабой сходимости последовательности $\left(x_{n}\right)$. Тогда для любого $\varepsilon>0$ найдется $n_{1}$ такое, что при $n \geqslant n_{1}$ выполняется

$$
\sum_{i=1}^{n}\left(F^{\prime}{ }_{x}\left(x\left(\xi_{i n}\right), \xi_{i n}\right), x\left(\xi_{i n}\right)-x_{i n}\right) \mu_{i n}<\varepsilon / 2 .
$$

Слабая сходимость дискретных мер $\mu_{n}$ к вероятностной мере $\mu$ вместе с условиями У1-У3 гарантирует при $n$, не меньших некоторого $n_{2}$, выполнение неравенства $\left|R_{n}\right|<\varepsilon / 2$. Лемма доказана.

С ле д с т в и е. При выполнении условий леммы 1 пара $\left\{f(x),\left(f_{n}\left(x_{n}\right)\right)\right\}$ дискретно непрерывна.

3 а м е ч а и е. Известно $\left[{ }^{2}\right]$, что условия У1-У4 гарантируют при $Q \cap S \neq \varnothing$ существование решения в задачах типа (1)-(3). Тем самым они существуют и в $(1 n)-(3 n)$ при $Q_{n} \cap S_{n} \neq \varnothing$ в случае любого $n \in N$.

Лемм а 2. Если выполнены условия У1-У4, то последовательность решений $\left(x_{n}{ }^{*}\right)$ задач $(1 n)-(3 n)$ дискретно слабо компактна и предельные точки этой последовательности удовлетворяют ограничениям (2) и (3) задачи (1)-(3). 
Д ок а з а те льс тв о. В силу условия У4 имеем

$$
\sum_{i=1}^{n}\left|x_{i n}^{*}\right|^{p} \mu_{i n} \leqslant \alpha_{k}^{-1} \sum_{i=1}^{n} a_{k}\left(\xi_{i n}\right) \mu_{i n}
$$

и, так как функция $a_{k}(\xi)$ интегрируема по Риману, то последовательность $\left\|x_{n}{ }^{*}\right\|_{n}(n \in N)$ ограничена. Согласно $\left[{ }^{4}\right]$ (с. 25 , теорема 4$)$, ограниченная последовательность $\left(x_{n}{ }^{*}\right)$ в силу рефлексивности пространства $L^{p}$ дискретно слабо компактна. Покажем, что предел $\bar{x}(\xi)$ последовательности $x_{n}{ }^{*}\left(n \in N^{\prime} \subset N\right)$ принадлежит множеству $X=Q \cap S$. Покажем сначала, что $\bar{x} \in Q$. Предположим противное, пусть $f_{j}(\bar{x})>0$ для некоторого $j$ из $\{1,2, \ldots, m\}$. В силу слабой дискретной полунепрерывности снизу пары $\left.\left\{f_{j}(x),\left(f_{j n} \mid x_{n}\right)\right)\right\}$ получим следующее противоречие

$$
0<f_{j}(\bar{x}) \leqslant \frac{\lim }{n \rightarrow \infty} f_{j n}\left(x_{n}^{*}\right) \leqslant 0 .
$$

Следовательно, $\bar{x} \in Q$.

Предположим теперь, что $\bar{x} \notin S$.

Пусть существует сфера $D \in B$ с положительной мерой $\mu(D)>0$ такая, что для любого $\xi \in D$ имеем

$$
h(\bar{x}(\xi), \xi) \geqslant \delta>0 .
$$

Тогда $\int \chi_{D}(\xi) h(\bar{x}(\xi), \xi) \mu(d \xi) \geqslant \delta \mu(D)$,

где

$$
\chi_{D}(\xi)=\left\{\begin{array}{l}
1, \text { если } \xi \in D, \\
0, \text { если } \xi \notin D .
\end{array}\right.
$$

Так как точки $x_{n}{ }^{*}\left(n \in N^{\prime}\right)$ удовлетворяют ограничениям задач $(1 n)-(3 n) \quad\left(n \in N^{\prime}\right)$, TO

$$
\sum_{i=1}^{n} \chi_{D}\left(\xi_{i n}\right) h\left(x_{i n}^{*}, \xi_{i n}\right) \mu_{i n} \leqslant 0 .
$$

Тогда

$$
\begin{gathered}
\delta \mu(D) \leqslant \int \chi_{D}(\xi) h(\bar{x}(\xi), \xi) \mu(d \xi) \leqslant \\
\leqslant \int \chi_{D}(\xi) h(\bar{x}(\xi), \xi) \mu(d \xi)-\sum_{i=1}^{n} \chi_{D}\left(\xi_{i n}\right) h\left(x_{i n}^{*}, \xi_{i n}\right) \mu_{i n}+ \\
+\sum_{i=1}^{n}\left(\chi_{D}\left(\xi_{i n}\right) h_{x}^{\prime}\left(\bar{x}\left(\xi_{i n}\right), \xi_{i n}\right), \bar{x}(\xi)-x_{i n}^{*}\right) \mu_{i n} .
\end{gathered}
$$

Условия У1-У3 гарантируют, что при $n$, не меньших некоторого $n_{1}$, оба слагаемых в последней сумме меньше $\delta \mu(D) \mid 4$. Полученное противоречие доказывает, что $\bar{x} \in S$. Следовательно, $\bar{x} \in X$, и лемма доказана.

Опираясь на леммы 1 и 2, сформулируем и докажем теорему о дискретной устойчивости задачи стохастического программирования с решающими функциями.

Т е о р е м а. Пусть выполнены следуюцие условия:

1) последовательность дискретных мер $\mu_{n}$ слабо сходится к вероятностной мере $\mu$, для которой выполнены условия У1 $и$ У2;

2) для функции $F_{k}(x, \xi), k=\overline{0, m+1}$, выполнены условия УЗ;

3) сущцествует индекс $j \in\{1,2, \ldots, m\}$, для которого выполнено усrовие $\mathrm{y} 4$;

4) существует точка $\tilde{x}=\tilde{x}(\xi)$ такая, что $f_{k}(\tilde{x})<0, k \in\{1,2, \ldots, m\}$ $u F_{m+1}(\tilde{x}(\xi), \xi)<0$ для почти всех $\xi \in G$. 


$$
\lim _{n \rightarrow \infty} f_{n}^{*}=f^{*},
$$

u предельные точки последовательности решений задач $(1 n)-(3 n)$ $(n \in N)$ являются оптимальными для задачи (1)-(3).

Док а з а те льст в о. По лемме $2 x_{n}{ }^{*}-\rightarrow \bar{x} \in X, \quad(n \in N)$. Тогда в силу слабой дискретной полунепрерывности снизу пары $\left\{f_{0},\left(f_{0 n}\right)\right\}$ имеем

$$
f^{*} \leqslant \bar{f}=f_{0}(\bar{x}) \leqslant \underline{\lim }_{n \rightarrow \infty} f_{0 n}\left(x_{n}^{*}\right)=\underline{\lim }_{n \rightarrow \infty} f_{n}^{*} .
$$

Докажем обратное неравенство. Пусть $x_{\varepsilon}$ является $\varepsilon$-решением задачи $(1)-(3)$, т. е. $f_{0}\left(x_{\varepsilon}\right) \leqslant f^{*}+\varepsilon / 2$. Это $\varepsilon$-решение существует ввиду условий 1) -4) теоремы, т. е.

$$
\begin{array}{cl}
\left|f^{*}-f_{0}\left(x_{\varepsilon}\right)\right|<\varepsilon / 2, \quad f_{k}\left(x_{\varepsilon}\right)<0, & k=\overline{1, m}, \\
F_{m+1}\left(x_{\varepsilon}(\xi), \xi\right)<0 \quad \text { при п. в. } & \xi \in G
\end{array}
$$

(строгое неравенство гарантировано условием 4)).

В силу условия У2 мы можем рассматривать $x_{\varepsilon}(\xi)$ как интегрируемую по Риману функцию. Тогда в силу условия У4, слабой сходимости дискретных мер $\mu_{n}$ к мере $\mu$ и вследствие непрерывности $F_{k}(x, \xi)$, $k=\overline{1, m}$, имеем

$$
\sum_{i=1}^{n} F_{k}\left(x_{e}\left(\xi_{i n}\right), \xi_{i n}\right) \mu_{i n}<0, \quad k=\overline{1, m .}
$$

Кроме того,

$$
F_{m+1}\left(x_{\varepsilon}\left(\xi_{i n}\right), \xi_{i n}\right)<0, \quad i=\overline{1, n} .
$$

Тогда при $n \geqslant n_{1}$ имеем для связывающих операторов $p_{n}(n \in N)$

$$
\begin{gathered}
\sum_{i=1}^{n} F_{k}\left(p_{n} x_{\varepsilon}, \xi_{i n}\right) \mu_{i n} \leqslant 0, \quad k=\overline{1, m}, \\
F_{m+1}\left(p_{n} x_{\varepsilon}, \xi_{i n}\right) \leqslant 0, \quad i=\overline{1, n},
\end{gathered}
$$

т. е. $p_{n} x_{\varepsilon} \in Q_{n} \cap S_{n}$ для всех $n$, начиная с некоторого $n_{1}$. Но $p_{n} x_{\varepsilon} \rightarrow x_{\varepsilon}$, и следовательно, в силу дискретной полунепрерывности сверху пары $\left\{f_{0},\left(f_{0 n}\right)\right\}$ имеем при $n$, не меньше некоторого $n_{2}$

$$
\begin{aligned}
& \bar{f}-f^{*} \leqslant\left|\bar{f}-f_{0}\left(x_{\varepsilon}\right)\right|+\left|f_{0}\left(x_{\varepsilon}\right)-f^{*}\right| \leqslant \\
\leqslant & \left|\lim _{n \rightarrow \infty} f_{0 n}\left(x_{n}^{*}\right)-f_{0}\left(x_{\varepsilon}\right)\right|+\left|f_{0}\left(x_{\varepsilon}\right)-f^{*}\right| \leqslant \\
\leqslant & \left|\lim _{n \rightarrow \infty} f_{0 n}\left(p_{n} x_{\varepsilon}\right)-f_{0}\left(x_{\varepsilon}\right)\right|+\left|f_{0}\left(x_{\varepsilon}\right)-f^{*}\right| .
\end{aligned}
$$

Последняя сумма меньше, чем $\varepsilon$ при $n \geqslant \max \left(n_{1}, n_{2}\right)$.

Покажем, что все дискретно предельные точки последовательности решений задач $(1 n)-(3 n)(n \in N)$ являются для задачи $(1)-(3)$ оптимальными.

Действительно, это верно потому, что пространства $L^{p}, l_{n}{ }^{p}(n \in N)$ обладают дискретным свойством Ефимова-Стечкина:

$$
x_{n} \longrightarrow \rightarrow x, \quad\left\|x_{n}\right\|_{n} \rightarrow\|x\| \Rightarrow x_{n} \rightarrow x .
$$

Этот факт легко доказывается исходя из свойства Ефимова-Стечкина в $L^{p}$ (см., напр., $\left[{ }^{14}\right]$, с. 293, теорема 1). Теорема доказана.

Основные результаты настоящей статьн анонсированы в $\left[{ }^{15}\right]$.

В заключение автор выражает признание Т. Тобиасу за полезные советы и замечания. 
1. Ермольев Ю. М. Методы стохастического программирования. М., «Наука», 1976.

2. Райк Э. Изв. АН ЭССР. Фнз. Матем., 21, № 3, 258-263 (1972).

3. Rockafellar, R. T., Wets, R. J.-B. Pacific J. Math., 62, № 1, 173-195 (1976).

4. Вайникко $\Gamma$. Анализ дискретизационных методов. Тарту, Изд-во Тартуск. ун-та, 1976.

5. Daniel, J. W. Math. Comput., 23, № 107, 573-581 (1969).

6. Карма О. Уч. зап. Тартуск. ун-та, вып. 448,99-106 (1978).

7. Васин В. В. Ж. вычисл. матем. и матем. фнз., 22, № 4, 824-839 (1982).

8. Васин В. В. Матем. заметки. 31, № 2, 269-280 (1982).

9. Römisch, W. Seminarber. Humboldt-Univ. Berlin. Sek. Math., № 39, $166-175$ (1982).

10. Kall, P., Stoyan, D. Math. Operationsforsch. Statist., Ser. Optimization, 13, № 3, $431-447$ (1982).

11. Kall, P. Z., Angew. Math. Phys., 30, № 2, $261-271$ (1979).

12. Olsen, P, Math. Programming Stud., 6, $111-124$ (1976).

13. Вайникко $\Gamma$. Компактная аппроксимация операторов и прнближенное решение уравнений. Тарту, Изд-во Тартуск. ун-та, 1970.

14. Канторович Л. В., Акилов Г. П. Функциональный анализ. М., «Наука», 1977.

15. Лenn P. В кн.: Методы решения нелинейных уравнений и задач оптимизации. III Симпоз. Докл. и сообщ. Таллин, 1984, 145-146.

Ннститут кибернетики

Академии наук Эстонской ССР

Поступнла в редакцию 5/II 1985

\section{R. $L E P P$}

\section{LAHENDUSFUNKTSIOONIDEGA OLDISE STOHHASTILISE PLANEERIMIS- ULESANDE DISKREETSE STABIILSUSE TINGIMUSED}

Integraalse funktsionaali minimeerimisülesanne integraalsetel kitsendustel ja kitsendustel, mille täitmist nõutakse peaaegu kindlasti, lähendatakse planeerimisülesannete jadaga Iōplikumõōtmelises ruumis. Eeldustel, mis nōuavad integreeritavate funktsioonide siledust ja jaotustiheduse integreerumist Riemanni mõttes, tõestatakse sellise lähendamise stabiilsus.

\section{R. $L E P P$}

\section{CONDITIONS FOR DISCRETE STABILITY OF A GENERAL STOCHASTIC PROGRAMMING PROBLEM WITH DECISION RULES}

Let $\xi=\xi(\omega)$ be a random vector with known distribution $\mu$. Consider the problem

$$
\min _{x(\xi)} \int F_{0}(x(\xi), \xi) \mu(d \xi)=f^{*}
$$

subject to constraints

$$
\int F_{k}(x(\xi), \xi) \mu(d \xi) \leqslant 0, \quad k=\overline{1, m},
$$

and $F_{m+1}(x(\xi), \xi) \leqslant 0$ for almost all $\xi \in G$.

In this paper we present discrete stability conditions for the problem (1)-(3) $L^{P}$-spaces.

Consider, instead of the problem (1)-(3), the following mathematical programming problem in finite-dimensional space:

$$
\begin{aligned}
& \min \sum_{i=1}^{n} F_{0}\left(x_{i n}, \xi_{i n}\right) \mu_{i n}=f_{n}^{*}, \\
& \sum_{i=1}^{n} F_{k}\left(x_{i n}, \xi_{i n}\right) \mu_{i n} \leqslant 0, \quad k=\overline{1, m}, \\
& F_{m+1}\left(x_{i n}, \xi_{i n}\right) \leqslant 0, \quad i=\overline{1, n} .
\end{aligned}
$$

Assuming that the weak convergence of the discrete measures $\left\{\left(\xi_{i n}, \mu_{i n}\right)\right\}$ to the probability measure $\mu$, which is supposed to have a Riemann integrable density, takes place, and some convexity and differentiability conditions for the functions $F_{k}(x, \xi)$, $k=\overline{0, m+1}$, hold, we prove that the problem (1)-(3) can be approximated by the sequence of problems $(\ln )-(3 n)$, i. e. $\lim _{n \rightarrow \infty} f_{n}^{*}=f^{*}$. 